\title{
Comment on "Explicit expressions for Green-Ampt (delta function diffusivity) infiltration rate and cumulative storage" by G. D. Salvucci and D. Entekhabi
}

\author{
D. A. Barry, ${ }^{1,2}$ J.-Y. Parlange, ${ }^{3}$ G. C. Sander, ${ }^{4}$ \\ and M. Sivapalan ${ }^{5}$
}

Salvucci and Entekhabi [1994] develop an approximation for the infiltration equation of Green and Ampt [1911]. Using dimensionless variables, the implicit form of the Green-Ampt equation is written

$$
t^{*}=I^{*}-\ln \left(1+I^{*}\right)
$$

In the notation of Salvucci and Entekhabi [1994] the dimensionless time and cumulative infiltration, respectively $t^{*}$ and $I^{*}$, are related to the actual time and cumulative infiltration, $t$ and $I$, by

$$
\begin{gathered}
I^{*}=I / \chi K_{s} \\
t^{*}=t / \chi .
\end{gathered}
$$

We note that $\chi$ is related to the sorptivity, $S$ (as affected by the ponded depth at the surface), via the expression: $S^{2}=2 K_{s}^{2} \chi$. The dimensionless form of the approximation of Salvucci and Entekhabi [1994, equation (10)], denoted $I_{\mathrm{SE}}^{*}$, is

$$
\begin{aligned}
I_{\mathrm{SE}}^{*}= & \left(1-\frac{2^{1 / 2}}{3}\right) t^{*}+\frac{2^{1 / 2}}{3}\left(t^{*}+t^{* 2}\right)^{1 / 2}+\frac{2^{1 / 2}-1}{3} \ln \left(1+t^{*}\right) \\
& +\frac{2^{1 / 2}}{3} \ln \left\{1+2\left[t^{*}+\left(t^{*}+t^{* 2}\right)^{1 / 2}\right]\right\} .
\end{aligned}
$$

The maximum relative error of $I_{\mathrm{SE}}^{*}$ is about $2.33 \%$ (Figure 1).

The purpose of this comment is to point out that simpler and much more accurate approximations for the Green-Ampt infiltration formula are already available.

Barry et al. [1993] showed that the solution to (1) can be written explicitly using the $W$ function [e.g., Fritsch et al., 1973]. Note that $W(x)$ is defined by solutions of

$$
W \exp (W)=x .
$$

Using this function, (1) can be rewritten as

$$
I^{*}=-1-W\left[-\exp \left(-1-t^{*}\right)\right] .
$$

\footnotetext{
${ }^{1}$ Department of Environmental Sciences and Engineering, University of North Carolina, Chapel Hill.

${ }^{2}$ On leave from Department of Environmental Engineering, Centre for Water Research, University of Western Australia, Nedlands.

${ }^{3}$ Department of Agricultural and Biological Engineering, Cornell University, Ithaca, New York.

${ }^{4}$ Faculty of Science and Technology, Griffith University, Nathan, Queensland, Australia.

${ }^{5}$ Department of Environmental Engineering, Centre for Water Research, University of Western Australia, Nedlands.

Copyright 1995 by the American Geophysical Union.

Paper number 95WR00092.

0043-1397/95/95WR-00092\$02.00
}

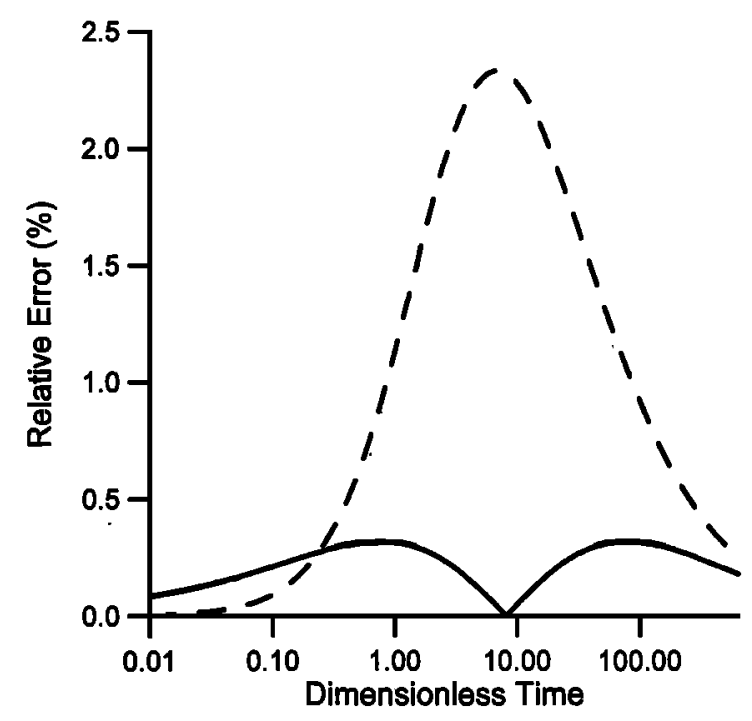

Figure 1. Magnitude of the relative error as a function of $t^{*}$ for $I_{1}^{*}$ (solid curve) and $I_{\mathrm{SE}}^{*}$ (dashed curve).

Clearly, once $W$ is approximated, an approximation for $I^{*}$ is obtained also. For infiltration, Bary et al. [1993] gave two approximations for $W$. The two approximations to the GreenAmpt infiltration formula are

$$
\begin{gathered}
I_{1}^{*}=t^{*}+\frac{2}{M_{1}}\left(1-\frac{1}{1+M_{1}\left(t^{*} / 2\right)^{1 / 2}}\right) \\
I_{2}^{*}=t^{*}+\frac{2}{m_{1}}\left[1-\frac{1}{\left.1+\frac{m_{1}\left(t^{*} / 2\right)^{1 / 2}}{1+m_{2} t^{*} \exp \left[m_{3}\left(t^{*}\right)^{1 / 2}\right]}\right]} .\right.
\end{gathered}
$$

The constants in (7) and (8) are $M_{1}=0.3205, m_{1}=0.3361$, $m_{2}=-0.0042$, and $m_{3}=-0.0201$. By contrast with the formula of Salvucci and Entekhabi [1994], the maximum relative errors of (7) and (8) are, respectively, $0.35 \%$ and $0.025 \%$. In particular, $I_{1}^{*}$ is markedly simpler than (4), while being an order of magnitude more accurate. Barry et al. [1993] pointed out that the form of the approximation used in (7) was first derived by Brutsaert [1977]. In Figure 1 the magnitude of the relative error for $I_{1}^{*}$ and $I_{\mathrm{SE}}^{*}$ is plotted. It is clear that over the entire range of $t^{*}$, a superior estimate is given by $I_{1}^{*}$, as this approximation minimizes the maximum error. Using $I_{2}^{*}$ is, as mentioned, an order of magnitude better again.

We note, in addition, that the Green-Ampt formula is also applicable for capillary rise. Barry et al. [1993] showed that in terms of the solution (6), this phenomenon corresponds to taking a different branch of the $W$ function. A simple approx- 
imation was developed for this case also (relative error, $<0.1 \%$ ).

Observe that relative error is not always a satisfactory criterion upon which to base approximations. The asymptotic (large $t^{*}$ ) expansion of the Green-Ampt equation is [e.g., Haverkamp et al., 1990]

$$
I^{*}=t^{*}+\ln \left(t^{*}\right)+\cdots
$$

None of the approximations presented here preserve the ln $\left(t^{*}\right)$ term on the right side of (9), and so the absolute value of the error will become unbounded as $t^{*} \rightarrow \infty$. One can, of course, adjust the approximations to ensure that the correct asymptotic behaviour is preserved. We do not carry out these operations here as they can be found in the recent paper by $D$. A. Barry et al. (Infiltration under ponded conditions, 4, An explicit infiltration formula, submitted to Soil Science, 1995). It is worth mentioning, however, that this latter work contains an explicit infiltration formula (derived from the implicit formula of Haverkamp et al. [1990]) that is valid for all soil types, i.e., for soils that lie between both the Green-Ampt and TalsmaParlange [Talsma and Parlange, 1972] limits. Remarkably, this formula is no more complicated than (4) or (8).

Finally, contrary to the implication contained in the title of Salvucci and Entekhabi [1994], the assumption of a delta function diffusivity leads not only to the infiltration formula for Green-Ampt soils, but also to the formula for Talsma-Parlange soils, as well as all soil types in between.

\section{References}

Barry, D. A., J.-Y. Parlange, G. C. Sander, and M. Sivapalan, A class of exact solutions for Richards' equation, J. Hydrol., 142(1-4), 2946, 1993.

Brutsaert, W., Vertical infiltration in dry soil, Water Resour. Res., 13(2), 363-368, 1977.

Fritsch, F. N., R. E. Shafer, and W. P. Crowley, Solution of the transcendental equation we $e^{w}=x$, Commun. Assoc. Comput. Mach., 16(2), 123-124, 1974.

Green, W. H., and G. A. Ampt, Studies in soil physics, 1, The flow of air and water through soils, J. Agric. Sci., 4, 1-24, 1911.

Haverkamp, R., J.-Y. Parlange, J. L. Starr, G. Schmitz, and C. Fuentes, Infiltration under ponded conditions, 3 , A predictive equation based on physical parameters, Soil Sci., 149, 292-300, 1990.

Salvucci, G. D., and D. Entekhabi, Explicit expressions for the GreenAmpt (delta function diffusivity) infiltration rate and cumulative storage, Water Resour. Res., 30(9), 2661-2663, 1994.

Talsma, T., and J.-Y. Parlange, One-dimensional vertical infiltration, Aust. J. Soil Res., 10, 143-150, 1972.

D. A. Barry and M. Sivapalan, Department of Environmental Engineering, Centre for Water Research, University of Western Australia, Nedlands, Western Australia 6907, Australia. (e-mail: barry@cwr. uwa.edu.au; sivapala@cwr.uwa.edu.au)

J.-Y. Parlange, Department of Agricultural and Biological Engineering, Cornell University, Ithaca, NY 14853-5701. (e-mail: jp58@ cornell.edu)

G. C. Sander, Faculty of Science and Technology, Griffith University, Nathan, Queensland 4111, Australia. (e-mail: G.Sander@sct. gu.edu.au)

(Received September 30, 1994; revised November 10, 1994; accepted January 9, 1995.) 